\title{
Self-assembly of magnetic biofunctional nanoparticles
}

\author{
Xiangcheng Sun ${ }^{\mathrm{a})}$ \\ Nuclear Engineering and Radiological Sciences, University of Michigan, Ann Arbor, Michigan 48109 \\ C. J. Thode \\ Chemistry Department, Penn State University, University Park, Pennsylvania 16802 \\ J. K. Mabry, J. W. Harrell, and D. E. Nikles \\ Center for Materials for Information Technology, The University of Alabama, Tuscaloosa, Alabama 35487
}

K. Sun and L. M. Wang

Nuclear Engineering and Radiological Sciences, University of Michigan, Ann Arbor, Michigan 48109

(Presented on 9 November 2004; published online 17 May 2005)

\begin{abstract}
Spherical, ferromagnetic FePt nanoparticles with a particle size of $3 \mathrm{~nm}$ were prepared by the simultaneous polyol reduction of $\mathrm{Fe}(\mathrm{acac})_{3}$ and $\mathrm{Pt}(\mathrm{acac})_{2}$ in phenyl ether in the presence of oleic acid and oleylamine. The oleic acid ligands can be replaced with 11-mercaptoundecanoic acid, giving particles that can be dispersed in water. Both x-ray diffraction and transmission electron microscopy indicated that FePt particles were not affected by ligands replacement. Dispersions of the FePt particles with 11-mercaptoundecanoic acid ligands and ammonium counter ions gave self-assembled films consisting of highly ordered hexagonal arrays of particles. () 2005 American Institute of Physics. [DOI: 10.1063/1.1846293]
\end{abstract}

\section{INTRODUCTION}

Nanobiotechnology is a promising new field incorporating nanocomposite materials designed for biological function. ${ }^{1-6}$ While biochemical applications are under heavy exploration, much of the diversity in nanoparticles systems is limited. Many challenges clearly remain before magnetic nanoparticles can achieve their full biological potential. ${ }^{1,2}$ Smaller and smarter magnetic iron oxide nanoparticles have been explored for biological applications both as tags in sensing and imaging. ${ }^{7,8}$ Recently, highly monodisperse magnetic nanoparticles, such as $\mathrm{Co}, \mathrm{FePt}$, and $\mathrm{Fe}_{2} \mathrm{O}_{3}$, have been synthesized in organic solvents. ${ }^{9-12}$ The advantage of this nonaqueous method is control over the particle size and shape with close to atomic layer precision, which strongly affects the chemical and physical properties of the nanoparticles. In almost all of these colloidal systems, a layer of surfactant molecules is essential to prevent aggregation. ${ }^{5}$ Often these surfactants are hydrophobic chains, causing the nanoparticles to be immiscible in the aqueous solutions. This makes biological applications difficult, due to the reaction conditions requiring hydrophobic solvents. To fulfill their potential for biological applications, it is therefore important to develop methods to transfer the particles into aqueous solutions. ${ }^{5}$ It is feasible to increase the aqueous phase dispersity of nanoparticles by modification of their soft surfaces, i.e., the surface-bound surfactant layers, of the nanoparticles. An effective strategy is to use ligand exchange with "water friendly" surfactants to increase the aqueous dispersity. Some pioneering works have established model systems of gold or silver particle transfer from organic solvent to aqueous phase through surface modification, improving the dispersity of nanoparticles in aqueous phase. ${ }^{13,14}$

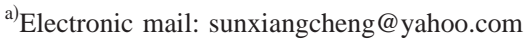

This work describes an effective way to increase the dispersity of oleic acid stabilized FePt nanoparticles in water. By employing thiol ligand chemistry, the stabilized FePt nanoparticles can be effectively transferred from hexane to aqueous solutions with the objective of biocompatibility.

\section{EXPERIMENTAL DETAILS}

\section{A. Synthesis of FePt particles}

This synthesis is based on a polyol reduction of $\mathrm{Fe}(\mathrm{acac})_{3}$ and $\mathrm{Pt}(\mathrm{acac})_{2}$ by 1,2-hexadecanediol. ${ }^{4}$ The reactions were carried out under an argon atmosphere in a $50-\mathrm{mL}$ three-necked round bottom flask equipped with magnetic stirring, a reflux condenser, a thermometer, and rubber septa. A solution of $\mathrm{Fe}(\mathrm{acac})_{3}$ and $\mathrm{Pt}(\mathrm{acac})_{2}$ and 1, 2-hexadecanediol in phenyl ether was heated to $80^{\circ} \mathrm{C}$. Then oleic acid and oleylamine were added via syringe. The solution was refluxed at $\sim 260^{\circ}$ for $30 \mathrm{~min}$. The reaction mixture was allowed to cool to room temperature, giving a dark dispersion. A $10-\mathrm{ml}$ ethanol was added to precipitate the particles, and the particles were isolated by centrifugation. The final FePt nanoparticles were redispersed in order to make thin films and perform liquid phase reactions using a small amount of dispersion solution containing hexane, octane, oleic acid, and oleylamine.

\section{B. FePt nanoparticles with dodecanethiol ligands}

The FePt particles with oleic acid ligands $(0.03 \mathrm{~g})$ were redispersed with a minimal amount of 50/50 hexane and octane. Following the addition of a $2.84 \mathrm{~mL}$ of 1-dodecanethiol, the solution was shaken for $\sim 1 \mathrm{~min}$. The 10 -mL centrifuge tube was filled with ethanol, sealed, and set aside to allow the particles to precipitate; the precipitated particles were mixed into a suspension in ethanol, centrifuge 


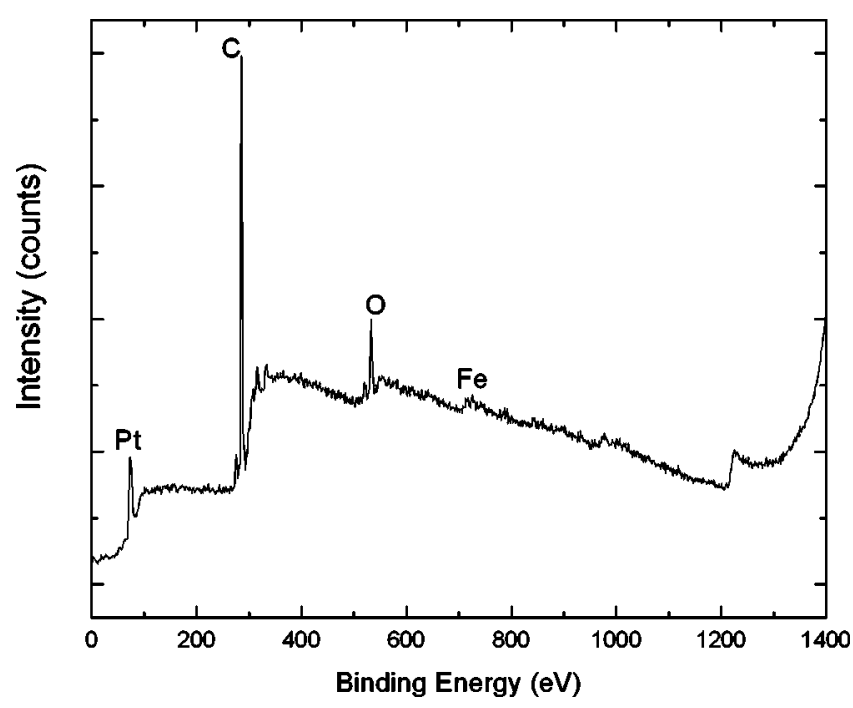

FIG. 1. The x-ray photoelectron spectrum of as-prepared FePt particles, metallic platinum, carbon, oxygen, and iron peaks was clearly presented.

in 10-min. intervals and the supernatant removed. This process was repeated until the supernatant was clear and colorless. The final resulting particles were almost black.

\section{FePt nanoparticles with 11-mercaptoundecanoic acid ligands}

The FePt nanoparticles with oleic acid ligands (0.04 g) were redispersed in a minimal amount of 50/50 hexane and octane in a $10-\mathrm{mL}$ centrifuge tube. To this dispersion was added a $5.0 \mathrm{~mL}$ of a saturated solution of 11mercaptoundecanoic acid in cyclohexanone. The dark brown solution was shaken for 1-2 min., whereupon the particles precipitated giving a clear grayish supernatant. The particles were rinsed first with cyclohexanone, then ethanol, and finally, acetone until the supernatant was clear. After each wash the particles were centrifuged for $10 \mathrm{~min}$ and the particles were redispersed with a minimal amount of cyclohexanone. The vial was filled with distilled water and a $5.0 \mathrm{~mL}$ of 1.48-M ammonium hydroxide $\left(\mathrm{NH}_{4} \mathrm{OH}\right)$ was then added. After shaking, a dark brown dispersion formed.

\section{Characterization}

A JEOL2010F scanning transmission electron microscope/transmission electron microscope (STEM/TEM) analytical electron microscope was performed to record high-angle annular dark-field (HAADF) images. All the TEM specimens were made by placing a drop of organic or water suspension of particles on a carbon-coated copper grid, and all the solvent solutions were allowed to evaporate at room temperature. X-ray diffraction curves were measured on a Rigaku thin-film x-ray diffractometer. The X-ray photoelectron spectra (XPS) were recorded on an AXIS 165 x-ray photoelectric spectrometer with an aluminum $\mathrm{x}$-ray source. The composition of the particles was determined using an energy dispersive $\mathrm{X}$-ray analysis (EDAX) detector on a Philips XL 30 scanning electron microscope.

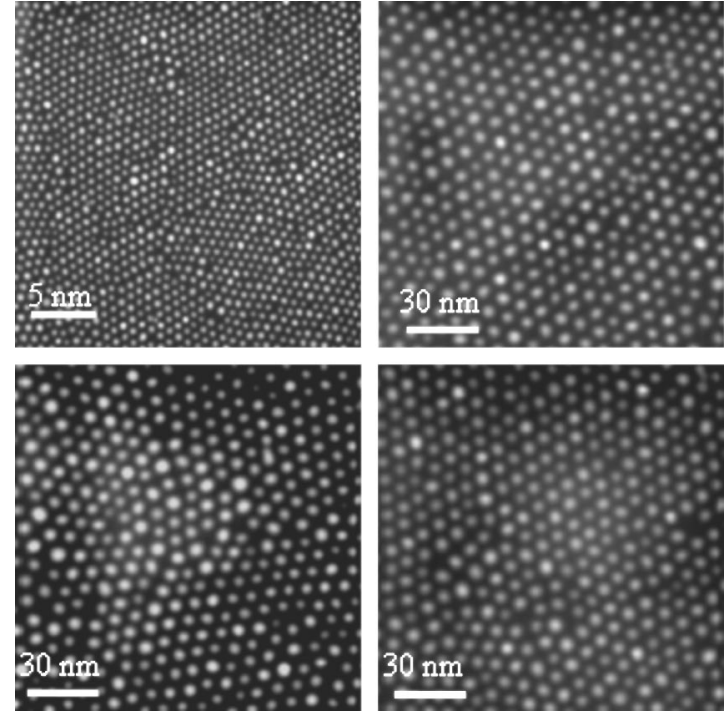

FIG. 2. 2D self-assembly arrays of as-prepared oleic acid stabilized FePt particles cast from hexane solutions.

\section{RESULTS AND DISCUSSION}

The as-prepared FePt particles had a coating of oleic acid which allowed dispersion of the particles in hydrocarbon solvents in order to cast thin films and perform solution phase chemistry. The particles have a chemically disordered face-centered cubic (fcc) structure ${ }^{9}$ and a particle size of approximately $3 \mathrm{~nm} .{ }^{6}$ The XPS of the as-prepared FePt nanoparticles, Fig. 1, showed the expected peaks due to metallic platinum, carbon, and oxygen. The iron peaks were weak and the binding energy was consistent with high valence iron. The HAADF images of two-dimensional (2D) self-assembly (or particle arrays) are shown in Fig. 2. The EDAX analysis confirms the presence of iron and platinum in varying compositional percentages between preparations. The general trend of the data suggests that FePt particles prefer to form in compositional ratios of 35:65 or 45:55 Fe:Pt.

It has been shown that the surfactants, oleic acid, and

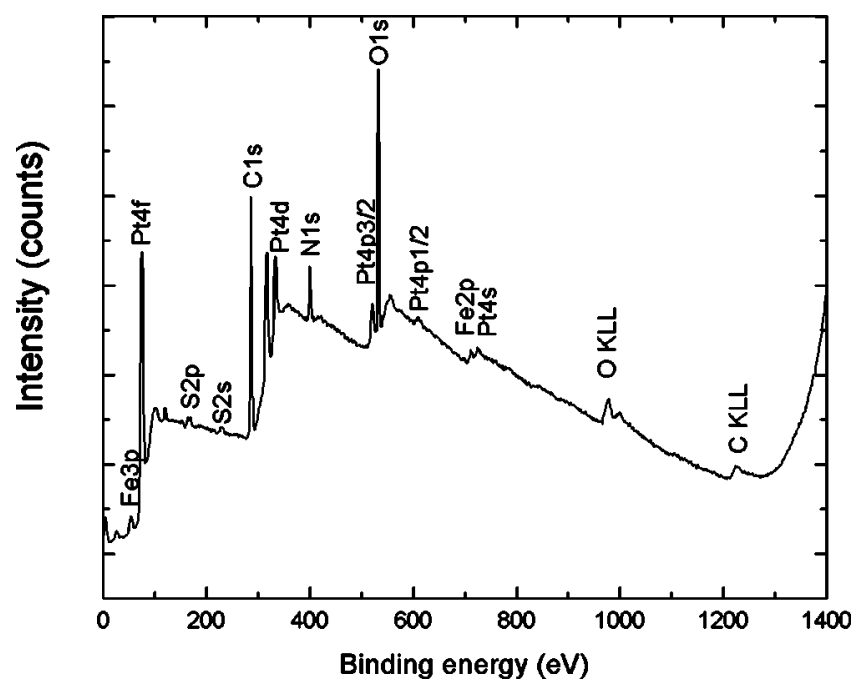

FIG. 3. X-ray photoelectron spectrum of FePt nanoparticles with 11mercaptoundecanoic acid ligands by using $\mathrm{NH}_{4} \mathrm{OH}$ on $\mathrm{Si}$ wafer. $\mathrm{N}$ and $\mathrm{S}$ were detected. 

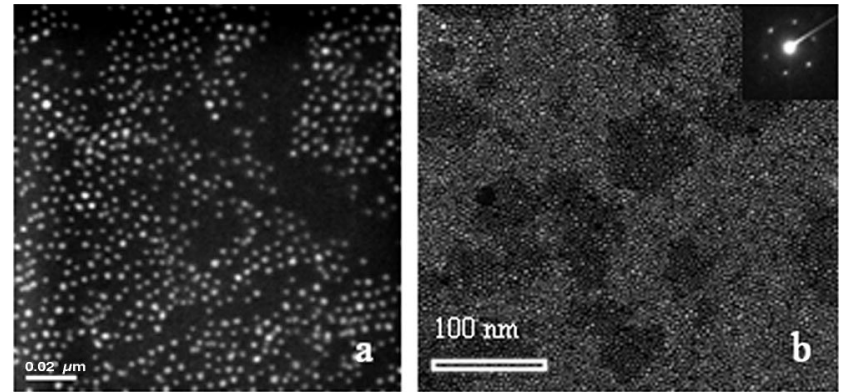

FIG. 4. (a) High-angle annular dark-field images for FePt particles having dodecanethiol ligands. (b) High-angle annular dark-field images for assemblies of FePt particles having 11-mercaptoundecanoic acid ligands with ammonium counter ions cast from aqueous dispersion. The inset selected area electron diffraction (SAED) pattern confirms the high degree of hexagonal particle positional ordering.

oleylamine can be replaced by organic compounds containing a thiol functional group. The thiol binds to the surface and creates an anchor for other molecules such as proteins. This is believed to happen because the aliphatic surfactants are in a rapid equilibrium with the bulk and the surface. Thiols, on the other hand, form covalent metal sulfur bonds, most distinctly with iron and, therefore, are found preferentially at the surface while the original surfactants are lost to the bulk solution. The thiol exchange assumed that the above-mentioned equilibrium will allow for a low hindrance for the thiol group to penetrate the surfactant layer. The particles that have undergone this procedure maintain their fcc lattice structure and retain their relative composition. This suggests that the thiolation procedure does not significantly degrade the particles. This is further confirmed in the XPS (Fig. 3) that both of the new peaks with the binding energies of 164 and $400 \mathrm{eV}$ are consistent with the presence of sulfur and nitrogen. Meanwhile, the distinctive $1-\mathrm{eV}$ shift of the S2 $p$ peak for sulfur bound to iron was obtained, as compared to $\mathrm{FeS}_{2}$ XPS. ${ }^{15}$ The platinum and iron are both present in the XPS as well, though the iron exists an oxidized form. The intensity of the oxygen peak at $533 \mathrm{eV}$ was decreased, indicating that most, if not all, the oleic acid had been replaced by dodecanethiol. What is interesting to note is that the particles remain monodisperse and continue to form $2 \mathrm{D}$ arrays, as confirmed by HAADF images shown in Fig. 4(a).

This thiolation procedure provides for a natural route to phase transition using omega thiol-functionalized long chain carboxylic acids. FePt nanoparticles can be dispersed in basic water when the oleic acid/oleylamine ligands were partially replaced with 11-mercaptoundecanoic acid. In fact, these aqueous particles retain their physical properties and are able to form excellent 2D or three-dimensional (3D) selfassembly arrays which are easily seen in HAADF images, as shown in Fig. 4(b). They also exhibit the same XPS peak shift for the $\mathrm{S} 2 p$ peak and are stable for up to a year in basic solution, demonstrating the preferentiality for the thiol functionality at the surface of the FePt particles.

\section{CONCLUSIONS}

A polyol reduction approach has been used to prepare 3-nm FePt nanoparticles. It has been revealed that these surfactants, oleic acid, and oleylamine can be replaced by organic compounds containing a thiol functional group. In which, by using thiol surface chemistry, these highly functionalized FePt particles can then be applied to many biological applications.

\section{ACKNOWLEDGMENTS}

One of the authors (J.M.) was a participant in our summer research experiences for teachers (RET) program during the summer of 2003 and 2004. Another author (C.J.T.) was supported by our summer research experiences for undergraduates. The Department of Chemistry administered both the RET and the REU programs with the support of the NSF Award No. CHE-0243883. This work has also been supported by the NSF Materials Research Science and Engineering Center Award No. DMR-9809423.

${ }^{1}$ P. Gold, Mater. Today 2, 36 (2004).

${ }^{2}$ Q. A. Pankhurt, J. Connolly, S. K. Jones, and J. Dobson, J. Phys. D 36, R167 (2003).

${ }^{3}$ H. Gu, P. L. Ho, K. W. T. Tsang, C. W. Yu, and B. Xu, Chem. Commun. (Cambridge) 15, 1966 (2003).

${ }^{4}$ L. E. Euliss, S. G. Grancharov, S. O'Brien, T. J. Deming, G. D. Stucky, C. B. Murray, and G. A. Held, Nano Lett. 3, 1489 (2003).

${ }^{5}$ Y. Wang, J. F. Wong, X. W. Teng, X. Z. Lin, and H. Yang, Nano Lett. 3, 1555 (2003).

${ }^{6}$ K. E. Elkins, T. S. Vedantam, J. P. Liu, H. Zeng, S. H. Sun, Y. Ding, and Z. L. Wang, Nano Lett. 3, 1647 (2003).

${ }^{7}$ J. M. Perez, T. O'Loughin, F. J. Simeone, R. Weissleder, and L. Josephson, J. Am. Chem. Soc. 124, 2856 (2002).

${ }^{8}$ M. Zhao, L. Josephson, Y. Tang, and R. Weissleder, Angew. Chem., Int. Ed. 42, 1375 (2003).

${ }^{9}$ S. H. Sun, C. B. Murray, D. Weller, L. Folks, and A. Moser, Science 287, 1989 (2000).

${ }^{10}$ S. H. Sun and H. Zeng, J. Am. Chem. Soc. 124, 8204 (2002).

${ }^{11}$ V. F. Puntes, K. M. Krishnan, and A. P. Alivisatos, Science 291, 2115 (2001).

${ }^{12}$ S. H. Sun and C. B. Murray, J. Appl. Phys. 85, 4325 (1999).

${ }^{13}$ D. I. Gittins and F. Caruso, Angew. Chem., Int. Ed. 40, 3001 (2001).

${ }^{14}$ A. Swami, A. Kumar, and M. Sastry, Langmuir 19, 1168 (2003).

${ }^{15}$ J. D. Batteas, T. S. Rufael, and C. M. Friend, Langmuir 15, 2391 (1999). 